\title{
Monitoring and Modeling Biofilms on Metal Surfaces
}

\author{
Oldham AL* \\ Biology Department, University of Texas, USA \\ *Corresponding author: Oldham AL, Assistant Professor of Biology, Biology Department, Odessa, Texas, USA
}

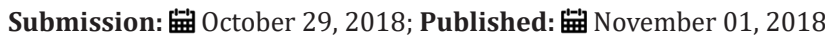

\section{Introduction}

Biofilms are aggregates of microorganisms that persist on a surface within a matrix of self-produced polysaccharides and other organic and inorganic matter. They can form on almost any material that comes into contact with water, and those present within marine systems and in the oil industry are made up of different types of microorganisms [1,2]. Because it is commonly accepted that some classes of microbes contribute to metal corrosion [3] and both industries rely on a primarily metal infrastructure, various laboratory studies include material from the industry partner (ie pipeline cutouts, biofilm material, production waters, etc) and at least one of the following approaches to monitor biofilm production and/or microbiologically-influenced corrosion

1. The use of scanning electron microscopy (SEM) coupled with energy dispersive X-ray (EDX) spectroscopy to visualize microorganisms within the biofilm and to determine the chemical composition of the biomass on a metal coupon's surface [3],

2. The use of molecular methods to remove biofilm material from a metal coupon's surface, extract DNA from the biofilm, and use Illumina seq analysis and quantitative PCR to identify and quantify the microorganisms within the biofilm $[1,2]$, and

3. The use of profilometry to assess changes in the roughness of the metal coupon's surface following the biofilm removal to determine if microbiologically-influenced corrosion has occurred and its extent $[4,5]$.

In addition to the above three approaches, production waters may also be cultured on selective media in an attempt to isolate and quantify organisms that have metabolic programs that are at odds with industrial surfaces or products. SEM and EDX analysis are an acceptable means of visualizing biofilm material and assessing corrosion products, but microscopy alone suffers from a major drawback. The biofilm material across the surface of the metal is not uniform. Rather, bacteria in the biofilm exist in microcolonies. In addition, the rates of microbiologically-influenced corrosion across the metal surface is not uniform. Rather, tiny pits can form in areas where the metal's crystalline structure may have been compromised. Because of this, a visual assessment of the biofilm should accompany at least one additional monitoring approach. Recovery of DNA from biofilm material and the subsequent sequence analysis is fairly robust, especially if the metagenome DNA is analyzed. This type of analysis allows the investigator to identify functional genes that could contribute to the microbiology in the system in addition to marker genes for microbial taxa classification. However, caution should be taken when drawing conclusions based on sequence analysis since DNA is among other organic molecules found within a biofilm. Meaning that the DNA sequence(s) of interest may be extracellular DNA and may not belong to vegetative microorganisms within the biofilm. Lastly, for assessing corrosion, profilometry is superior to SEM. Taken together, the above approaches are used by laboratories that collaborate with industry partners and usually more than one approach is included with the partner's monitoring and mitigation protocols.

Routine maintenance to limit biomass accumulation in industrial settings usually includes the application of biocides or antifouling coatings reviewed in [6] and the physical removal of the dead organic matter/biofilm from the metal surface [7]. While coupling these methods is common - killing the microbes and physically scraping the dead biomass away, additional methods that are more environmentally friendly are being explored in research laboratories. These include

1. The application of electrochemical current to the metal to inhibit the attachment of bacteria to the surface reviewed in [6], and

2. More recently, targeting bacterial quorum sensing systems. Chemical inhibitors of quorum sensing function to limit the transition of a microbe from a free-floating lifestyle to that of a biofilm constituent rather than killing it. This approach has gained appeal since it has a more desirable outcome reviewed in [8]. The inhibitors alter the life style of the organism, allowing it to remain in the liquid phase where it can be washed out of the system, rather than joining the solid phase where it would remain in the system indefinitely

Modeling the conditions of an industrial complex is of the utmost importance when the goal of the study is to make recommendations as to best practices for biofilm mitigation. To model biofilms in a laboratory setting, investigators require metal alloys of 
the same composition as that from the affected facility and water samples or biofilm material which contains the source of the resident microbes. In some cases, pipeline "cookies" are removed from dead legs or small metal coupons of the same composition may be purchased commercially from a company such as Metal Samples (Alabama Specialty Products Inc.) [4]. Organisms that are isolated from the water/biofilm and can be cultured in the lab are usually screened for biofilm formation. And in some cases, the isolates are screened for the ability to utilize components of the industry's product, be it fuel blends or biocides [5]. In addition, incubation conditions should be similar to that in the industrial complex. For example, bacteria isolated from the ballast tanks of ships that have sat for eight months undeployed are cultured in the lab under static conditions. Whereas, isolates from the production fluids that flow through an oil production facility are cultured under continuous flow conditions. These results from these types of studies are important to both oil production and in marine systems.

\section{Conclusion}

The methodology used to monitor industrial biofilms requires a varied approach. While this article highlights a handful of methods, many more are being explored and much knowledge has been gained over the past few decades as technologies continue to improve.

\section{References}

1. Oldham AL, Drilling HS, Stamps BW, Stevenson BS, Duncan KE (2012) Automated DNA extraction platforms offer solutions to challenges of assessing microbial biofouling in oil production facilities. AMB Express 2(1): 60.

2. Oldham AL, Steinberg MK, Duncan KE, Makama Z, Beech I (2017) Molecular methods resolve the bacterial composition of natural marine biofilms on galvanically coupled stainless steel cathodes. J Ind Microbiol Biotechnol 44(2): 167-180.

3. Liduino VS, Lutterbach MTS, Sérvulo EFC (2018) Biofilm activity on corrosion of API 5L X65 steel weld bead. Colloids and Surfaces B: Biointerfaces 172: 43-50.

4. Lenhart TR, Duncan KE, Beech IB, Sunner JA, Smith W, et al. (2014) Identification and characterization of microbial biofilm communities associated with corroded oil pipeline surfaces. Biofouling 30(7): 823835.

5. Liang R, Aydin E, Le Borgne S, Sunner J, Duncan KE, et al. (2018) Anaerobic biodegradation of biofuels and their impact on the corrosion of a Cu-Ni alloy in marine environments. Chemosphere 195: 427-436.

6. Sultana ST, Babauta JT, Beyenal H (2015) Electrochemical biofilm control: A review. Biofouling 31(9-10): 745-758.

7. Quarini J, Shire S (2007) A Review of fluid-driven pipeline pigs and their applications. Proc Inst Mech Eng Part E J Process Mech Eng 221: 1-10.

8. Algburi A, Comito N, Kashtanov D, Dicks LMT, Chikindas ML (2017) Control of biofilm formation: Antibiotics and Beyond. Appl Environ Microbiol 83(3).

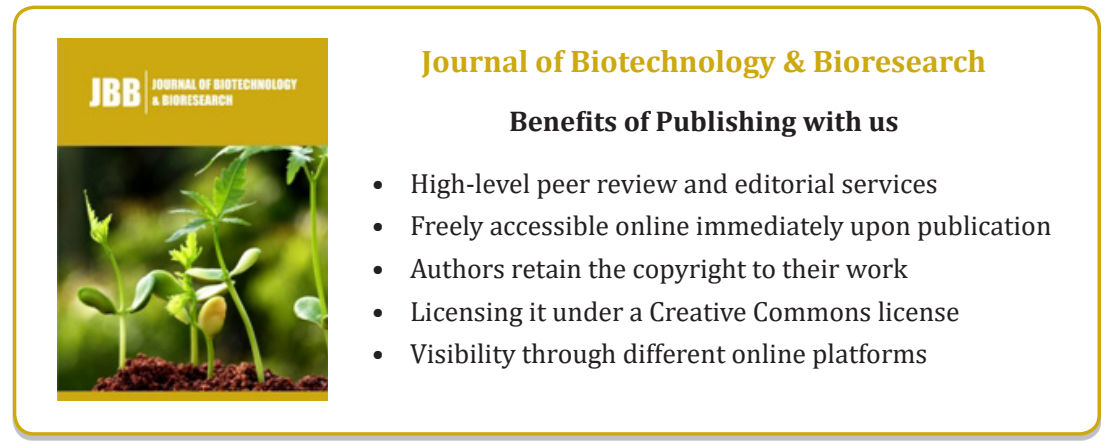

